\title{
POLYMERASE CHAIN REACTION: A POWERFUL DIAGNOSTIC AND RESEARCH TOOL
}

\author{
Dr. Kanu Jain ${ }^{1}$, Dr. Monica Mehendiratta ${ }^{2}$, Dr. Deepti Garg Jindal ${ }^{3}$, Dr. Mohit Bansal ${ }^{4}$ \\ ${ }^{1}$ Senior Lecturer, Sudha Rustagi Dental College and Research Institute, Faridabad, Haryana (India) \\ ${ }^{2}$ Reader, Inderprastha Dental College and Hospital, Ghaziabad, Uttar Pradesh (India) \\ ${ }^{3}$ Reader, Dept. of Oral and Maxillofacial Pathology, Bhojia Dental College, Distt. Solan, Himachal Pradesh (India) \\ ${ }^{4}$ Senior Resident, Department of Community Dentistry, Oral Health Sciences Center, PGIMER, Chandigarh (India) \\ Corresponding Author: \\ ${ }^{1}$ Mobile: 919818887636 Email: drkanupriyajain@gmail.com
}

\begin{abstract}
Received :
$1^{\text {st }}$ April, 2013

Accepted:

$16^{\text {th }}$ June, 2013

Available online:

$25^{\text {th }}$ August, 2013

To fully understand cellular processes, scientists often examine events at the level of nucleic acids and protein molecules. These studies are complicated by the fact that cells have miniscule amounts of molecules of interest which are too small to be seen. So we require molecular tools to visualize these molecules. Polymerase chain reaction is one such technique widely used in molecular biology and produce quantities that are sufficient to study and visualize. With the advancement in this technique, it has revolutionized the field of research and diagnosis. In this article, we present a review on the principle, basic technique, applications, limitations and recent advances of Polymerase Chain Reaction.
\end{abstract}

Keywords: Polymerase Chain Reaction, Deoxyribose Nucleic Acid, DNA Polymerase

\section{INTRODUCTION}

The polymerase chain reaction (PCR) is a rapid laboratory technique for "amplifying" a specific Deoxyribose nucleic acid (DNA) sequence. It is a process whereby a strand of deoxyribonucleic acid can be cloned million of times, within a few hours. PCR derives its name from one of its components, an enzyme, DNA polymerase used to amplify a piece of DNA by in-vitro enzymatic replication. It is called "chain" because the products of the first reaction become substrates of the following one, and so on. As PCR progresses, the DNA thus generated is itself used as template for replication. This sets in motion a chain reaction in which the DNA template is exponentially amplified.PCR uses few basic components to make large numbers of copies of a specific DNA fragment in a test-tube. Thus PCR is called a DNA-photocopier with tremendous power and versatility for DNA manipulation and analysis. PCR is extremely efficient and sensitive; it can make millions or billions of copies of any specific sequence of DNA, even when the sequence is in a complex mixture. Because of this power, researchers can use it to amplify sequences even if they have only a minute amount of DNA. A single hair root, or a microscopic blood stain left at a crime scene, for example, contains ample DNA for PCR. The process can be used to make prenatal diagnosis of genetic diseases and to identify an individual by analysis of a single tissue cell.

\section{HISTORICAL EVOLUTION}

Dr. Hargobind Khorana and colleagues described a technique for replicating a region of duplex DNA using two DNA synthesis primers. ${ }^{1}$

In 1983, Dr. Kary Mullis invented and developed PCR for which he received Nobel Prize in Chemistry in 1993. ${ }^{2,3} \mathrm{He}$ discovered that by harnessing the primer component of molecular reproduction technology, a target DNA of interest could be amplified exponentially.

\section{PRINCIPLE OF PCR}

The simplest concept of PCR relies on repeated synthesis of the targeted DNA by enzyme DNA polymerase. There are three steps needed to achieve this which are carried out rapidly and in succession at different and controlled temperatures, allowing for the doubling of the amount of DNA after each cycle. ${ }^{4}$ The three steps are denaturation, annealing and 
extension which are further discussed in PCR reaction.

\section{COMPONENTS OF PCR}

1. Target DNA - Itserves as target for PCR. It contains the sequence to be amplified. It is also known as Template DNA. It is obtained by isolating nucleic acid from samples.

2. Pair of Primers / Oligonucleotide Primers - These are short pieces of DNA (15-30 nucleotides in length) that bind to Template DNA allowing enzyme DNA Polymerase to initiate incorporation of deoxynucleotides. These primers are used to start synthesis of new strands of DNA.

3. Deoxynucleotidephosphates - They act as DNA building blocks. It is important that same concentration of each deoxynucleotide be used to prevent misincorporation of incorrect deoxynucleotides.

4. Thermostable DNA polymerase - It is the enzyme that catalyses PCR Reaction. In originally described method of PCR, a fragment from E.coli was used as DNA polymerase which had to be added after every step because it denatures at temperatures which were used in PCR Cycle. In 1988, a heat stable DNA polymerase from thermophilic bacterium, Thermus aquaticus (Taq) was used which did not have to be added after every step. $5,6,7$

5. Magnesium ions - They are usually present in the form of Magnesium Chloride and act as cofactor for enzyme and thus, required for proper functioning of DNA polymerase.

6. Buffer Solution - It maintains ph of the reaction to generate proper conditions for activity of enzyme. It is composed of Tris Hydrochloric acid, a salt, potassium chloride at a $\mathrm{pH}$ of 8.3.

7. Thermal Cycler - It is an instrument in which cycles of PCR reaction are carried out. It heats and cools PCR Cycle steps.

\section{DNA ISOLATION}

High quality genomic DNA can be obtained from variety of organisms including human beings, small mammals, plants, yeasts and bacteria. For humans, many different types of clinical samples have been used for PCR analysis including blood, saliva, sputum, semen and single hair. For each organism or tissue source, a specific procedure is provided for releasing free chromosomal DNA from its cellular or nuclear location. A standard protocol for DNA isolation was given by Wolff et al in 1992 which is widely used and can be used with any suspension of mammalian cells. ${ }^{8,9}$ These days commercial kits are available which quickly isolate DNA for various PCR based applications. ${ }^{10,11}$

\section{PCR REACTION}

Isolated DNA alongwith other components, Taq DNA Polymerse, buffers, nucleoside triphosphates and DNA primers is placed in thin walled PCR tubes and these tubes are then placed in the PCR Thermal cycler. The primers are $15-30$ nucleotides long and are complimentary in sequence to the ends of target DNA. Taq Polymerase is derived from hotsprings bacteria and controls the intensity of PCR. A PCR reaction lasts several hours and typically consists of about 25 - 35 repeating cycles. A cycle begins by heating the reaction mixture to $95^{\circ} \mathrm{C}$. The heat denatures DNA, break the hydrogen bonds and separate the two strands. After this, temperature is reduced to around $60^{\circ} \mathrm{C}$ so that primers can form hydrogen bonds or anneal over their complimentary sequences in target DNA. The primers and target DNA follow the base pairing rules so that adenine binds to thymine and cytosine binds to guanine. In the next phase, temperature is raised to about $70^{\circ} \mathrm{C}$. Taq Polymerase functions optimally at this temperature and begins polymerization adding nucleotides to 3 ' prime end, each primer attach to DNA strand. After one complete cycle, there are two copies of double stranded target DNA. PCR reaction mixture contains many copies of primers and in abundance of free nucleotides to perform many additional cycles. After the second cycle, there are four copies of the target DNA. After the cycle three is finished, there are eight copies of the double stranded target sequence. As the number of cycles increase, the product consist of greater proportions or fragments with just the target DNA. After four cycles, half of the fragments just consist of target DNA and half of the fragments also contain flanking DNA. With each additional cycle, the number of 
copies of target sequence doubles and at the end of cycle twenty five, there are more than thirty three million copies.

\section{PCR ANALYSIS}

After DNA is amplified, PCR tube is removed from Thermal Cycler and subjected to analysis to interpret the levels of product generated for example in a diagnostic context. The simplest and most direct method to analyze DNA is Gel Electrophoresis. In this technique, by placing the molecules in wells in the gel and applying an electric current, the molecules will move through the matrix at different rates usually determined by mass. In the case of DNA, the direction of migration is from negative electrode to positive electrode is due to naturally occurring negative charge carried by their sugar phosphate backbone. After the electrophoresis is complete, the molecules in the gel can be stained to make them visible. If the DNA molecules fluoresce under UV light, a photograph can be taken of the gel under UV lighting condition.

\section{APPLICATIONS OF PCR}

Many different types of clinical samples have been used for PCR analysis, including blood, saliva, sputum, semen and single hair. PCR has applications in the following mentioned fields: ${ }^{12,13,14}$

1. Microbiology: The use of PCR has revolutionized the diagnosis and study of infectious diseases and malignancies associated with micro-organisms. PCRbased systems to detect the etiologic agents of disease directly from clinical samples, without the need for culture, have been useful in rapid detection of unculturable or fastidious microorganisms. Additionally, sequence analysis of amplified microbial DNA allows for identification and better characterization of the pathogen. ${ }^{15}$ It overcomes many of the problems associated with culture methods and in some cases has replaced traditional pathogen identification methods.The DNA or RNA of an infectious organism can be detected in test material even when the organism number is low or is slowly growing, or when the infectious agent is in material that is not suitable for culture. ${ }^{16}$

2. Human genetics: Polymerase chain reaction plays an important role in the identification of chromosome disorders and hereditary diseases. These include
Cystic fibrosis, Gaucher's disease, $\alpha-1$ antitrypsin deficiency, hemophilia and sickle cell anemia, thalassemia.PCR can also be used to analyze fetal DNA for Aneuploidy, Trisomy 21, Turner's syndrome, Klinefelter's syndrome as well as for sex determination.

3. Forensic pathology: It has employed PCR for a variety of situations, including the identification of mutilated or decomposed human tissues, for sex determination and for disputed paternity cases. ${ }^{13}$ DNA fingerprinting is based on the identification of VNTRs-short, repeating DNA nucleotide sequences that are located throughout the human genome. This process has proved to be an important tool in the identification of criminals.

4. Tumour biology: PCR has revolutionized the study of cancer and provided greater insights into the pathobiology of neoplasia. (13) PCR has been used to detect mutations in cancer associated oncogenes (example K-ras, N-ras), tumor suppressor genes (p53, p16), monoclonality in B and T-cell lymphomas, chromosome translocations such as the Philadelphia chromosome $t(14 ; 18)$ in chronic myelogenous leukemia, and minimal residual neoplastic disease, as well as the study of genetic alterations in formalin-fixed tissues. Since PCR is ideally suited to the study of low numbers of unique DNA fragments, it has been applied to the detection of malignant cells in urine, sputum and saliva.

5. Cloning genes: Cloning a gene, describes the process of isolating a gene from one organism and then inserting it into another genetically modified organism (GMO). ${ }^{17}$ PCR is often used to amplify the gene, which can then be inserted into a vector such as a plasmid. The DNA can then be transferred into the GMO where the gene and its product can be studied more closely. Expressing a cloned gene can also be a way of mass-producing useful proteins.

6. Comparison of gene expression: Researchers have used traditional PCR as a way to estimate changes in the amount of a gene's expression. Once RNA is isolated it can be reverse transcribed back into DNA (complementary DNA). When the products of the PCR reaction are run on an agarose gel, a band corresponding to a gene, will appear larger on the gel. 
By running samples of amplified cDNA from differently treated organisms one can get a general idea of which sample expressed more of the gene of interest.

8. Mutagenesis: Mutagenesis is a way of making changes to the sequence of nucleotides in the DNA. There are situations in which one is interested in mutated (changed) copies of a given DNA strand, for example, when trying to assess the function of a gene or in in-vitro protein evolution. One application of mutagenesis is to analyze structure-function relationships of a protein. By randomly altering a DNA sequence, one can compare the resulting protein with the original and determine the function of each part of the protein. ${ }^{17}$

\section{LIMITATIONS OF PCR}

Although PCR is a powerful method to increase the amount of a gene of interest in vitro, it has a number of important limitations. ${ }^{12}$

1. Difficulties can be encountered when studying small quantities of DNA, since the ingredients necessary for PCR (oligonucleotide primers, dNTPs and Taq DNA polymerase) may be exhausted before sufficient target is produced.

2. The specificity of the reaction may be limited and depends on many complex, interrelated factors including the oligonucleotide-primer size, annealing temperature and buffer salt concentration.

3. Long DNA fragments (> 300 base pairs) are difficult to amplify when the starting material is degraded, such as that obtained following formalin fixation.

4. A major limitation of PCR is the susceptibility of the process to contamination, particularly in experiments intended to detect rare DNA sequences. With clean laboratory techniques and with appropriate sample controls, this problem can be overcome.

5. Finally on occasion, the Taq DNA polymerase may produce nucleotide addition errors, since the enzyme has no 'proof-reading properties.'
6. The tissue fixative $4 \%$ neutral buffered formalin causes cross-linking and nicks the DNA. Although proteolytic digestion of tissues produces large quantities of DNA, most is fragmented, thus limiting the size of the target area of the gene that can be subjected to PCR. Precipitating fixatives such as ethanol and acetone do not cross link or shear the DNA and therefore produce more consistent PCR results. Longer fixative times (more than 24 hours) may also adversely affect the quality of the DNA extracted from routinely processed tissues and thereby reduce PCR efficiency.

7. The counter-stain used for tissue visualization, before DNA extraction, can also affect the quality of the reaction. For example methyl green and neutral fast red counter stains produce PCR efficiency comparable to that obtained with unstained sections. By contrast, hematoxylin often produces significantly poorer PCR results than other stains and may be due to the binding of hematoxylin to DNA phosphate groups and to increased resistance to protease digestion.

\section{PCR OPTIMIZATION}

Non optimal PCR conditions can lead to numerous problems including low yield of the desired PCR product, inability to amplify low numbers of DNA template molecules or amplification of undesirable PCR products (non specific amplification). Some of the common parameters that might need to be optimized to increase sensitivity of detection or amplification specificity are: ${ }^{18,19}$

a) Selecting new primers

b) Modifying thermal cycling conditions

c) Altering the $\mathrm{Mg}^{2+}$ concentration

d) Altering the $\mathrm{pH}$

e) Altering other reaction components

\section{MODIFIED PCR TECHNIQUES AND RECENT ADVANCES}

1. Inverse PCR:

A method used to allow PCR when only one internal sequence is known. This is especially useful in identifying flanking sequences to various genomic inserts. This involves a series of DNA digestions and self ligation, resulting in known sequences at either end of the unknown sequence. ${ }^{13}$ 


\section{Ligation-mediated PCR:}

This method uses small DNA linkers ligated to the DNA of interest and multiple primers annealing to the DNA linkers; it has been used for DNA sequencing, genome walking, and DNA foot printing. ${ }^{13}$

\section{Methylation-specific PCR (MSP):}

It was developed by Stephen Baylin and Jim Herman at the Johns Hopkins School of Medicine and is used to detect methylation of $\mathrm{CpG}$ islands in genomic DNA. DNA is first treated with sodium bisulfite, which converts unmethylated cytosine bases to uracil, which is recognized by PCR primers as thymine. ${ }^{13}$ Two PCRs are then carried out on the modified DNA, using primer sets identical except at any $\mathrm{CpG}$ islands within the primer sequences. At these points, one primer set recognizes DNA with cytosines to amplify methylated DNA, and one set recognizes DNA with uracil or thymine to amplify unmethylated DNA. MSP using qPCR can also be performed to obtain quantitative rather than qualitative information about methylation.

\section{Multiplex-PCR:}

The use of multiple, unique primer sets within a single PCR mixture to produce amplicons of varying sizes specific to different DNA sequences. By targeting multiple genes at once, additional information may be gained from a single test run that otherwise would require several times the reagents and more time to perform. Annealing temperatures for each of the primer sets must be optimized to work correctly within a single reaction, and amplicon sizes, i.e., their base pair length, should be different enough to form distinct bands when visualized by gel electrophoresis. It can help in detecting different pathogens in a single specimen. ${ }^{20,21}$

\section{Multiplex Ligation-dependent Probe Amplification (MLPA):}

It permits multiple targets to be amplified with only a single primer pair, thus avoiding the resolution limitations of multiplex PCR. ${ }^{13}$

\section{Nested PCR:}

It increases the specificity of DNA amplification, by reducing background due to non-specific amplification of DNA. Two sets of primers are being used in two successive PCRs. ${ }^{22}$ In the first reaction, one pair of primers is used to generate DNA products, which besides the intended target, may still consist of non-specifically amplified DNA fragments. The products are then used in a second PCR with a set of primers whose binding sites are completely or partially different from and located 3' of each of the primers used in the first reaction. Nested PCR is often a rapid ${ }^{23}$ and more successful in specifically amplifying long DNA fragments than conventional PCR, but it requires more detailed knowledge of the target sequences.

\section{Quantitative PCR (Q-PCR):}

It is used to measure the quantity of a PCR product (preferably real-time). It is the method of choice to quantitatively measure starting amounts of DNA, cDNA or RNA. $^{13}$ Q-PCR is commonly used to determine whether a DNA sequence is present in a sample and the number of its copies in the sample. The method with currently the highest level of accuracy is Quantitative real-time PCR. It is often confusingly known as RT-PCR (Real Time PCR) or RQ-PCR. RT-PCR commonly refers to reverse transcription PCR which is often used in conjunction with Q-PCR. QRT-PCR methods use fluorescent dyes, such as SYBR Green, or fluorophorecontaining DNA probes, such as TaqMan, to measure the amount of amplified product in real time. ${ }^{24,25}$

\section{Reverse Transcription PCR (RT-PCR):}

It is a method used to amplify, isolate or identify a known sequence from a cellular or tissue RNA. The PCR is preceded by a reaction using reverse transcriptase to convert RNA to cDNA. RT-PCR is widely used in expression profiling, to determine the expression of a gene or to identify the sequence of an RNA transcript, including transcription start and termination sites and, if the genomic DNA sequence of a gene is known, to map the location of exons and introns in the gene. The 5' end of a gene (corresponding to the transcription start site) is typically identified by an RT-PCR method, named RACE-PCR, short for Rapid Amplification of cDNA ends. ${ }^{13}$ This type of PCR is useful for diagnosis of RNA viruses, as well as evaluation of antimicrobial therapy. ${ }^{26}$ 


\section{Real time PCR}

In this, products are analysed at the end of each PCR cycle. It makes use of fluorescent labels to monitor progress ofPCR amplification. ${ }^{24}$ Two major methods are double-stranded DNA binding agents (eg SYBR Green) and Sequence Specific Probes (eg Taqman, Mol Beacons). ${ }^{24,27}$

\section{Touchdown PCR}

Touchdown PCR is a variant of PCR that reduces nonspecific primer annealing by lowering of annealing temperature between cycles. ${ }^{13}$

\section{Colony PCR}

It is an extremely simple and effective procedure which is established for both gram negative and gram positive bacteria, yeasts and microalgae. ${ }^{28,29}$ Bacterial clones can be screened for the correct ligation products. Selected colonies are picked with a sterile toothpick from a agarose plate and dabed into the master mix or sterile water. Primers (and the master mix) are added-the PCR protocol has to be started with extended time at $95^{\circ} \mathrm{C} .^{13,29}$

\section{Allele-specific PCR}

An allele specific PCR was developed to detect single nucleotide polymorphisms (SNPs) (single-base differences in DNA) in human DNA samples. ${ }^{30}$ It is a diagnostic or cloning technique based on SNPs. (13) It requires prior knowledge of a DNA sequence, including differences between alleles, and uses primers whose $3^{\prime}$ ends encompass the SNP. ${ }^{30}$ PCR amplification under stringent conditions is much less efficient in the presence of a mismatch between template and primer, so successful amplification with an SNP-specific primer signals presence of the specific SNP in a sequence.

\section{Assembly PCR or Polymerase Cycling Assembly (PCA)}

It refers toartificial synthesis of longDNA sequences by performing PCR on a pool of long oligonucleotides with short overlapping segments. ${ }^{31}$ The oligonucleotides alternate between sense and antisense directions and the overlapping segments determine the order of the PCR fragments, thereby selectively producing the final long DNA product.

\section{Asymmetric PCR}

It preferentially amplifies one DNA strand in a double-stranded DNA template. ${ }^{13}$ It is used in sequencing and hybridization probing where amplification of only one of the two complementary strands is required. It is a cost effective method for production of single stranded DNA. ${ }^{32}$ PCR is carried out as usual, but with a great excess of the primer for the strand targeted for amplification. This method, however, is difficult to optimize, often inefficient and tends to promote non specific amplification. ${ }^{33} \mathrm{~A}$ recent modification on this process, known as LinearAfter-The-Exponential-PCR (LATE-PCR) solves these problems.

\section{LATE-PCR}

It is a method similar to asymmetric PCR in the use of primers at different concentrations, but with novel design criteria to ensure high efficiency and specificity. ${ }^{34}$ It uses a limiting primer with a higher meltingtemperature than the excess primer to maintainreaction efficiency as the limiting primerconcentration decreases mid-reaction.

\section{Dial-out PCR}

It isa highly parallel method for retrieving accurate DNA molecules for gene synthesis. A complex library of DNA molecules is modified with unique flanking tags before massively parallel sequencing. Tag-directed primers then enable the retrieval of molecules with desired sequences by PCR. ${ }^{35}$

\section{Helicase-dependent amplification}

It issimilar to traditional PCR, but uses a constant temperature rather than cycling through denaturation and annealing/extension cycles. This method utilizes DNA helicase, an enzyme that unwinds DNA, in place of thermal denaturation which generates single stranded templates for primer hybridization and subsequent primer extension by a DNA polymerase. ${ }^{36}$

\section{Hot start PCR}

It is a technique that reduces nonspecific amplification during the initial set up stages of the PCR. It entails the withholding of at least one reagent from reaction mixture until the reaction tube temperature has reached $60-80^{\circ} \mathrm{C}$. It may be performed manually by heating the reaction components to the denaturation temperature (e.g., $95^{\circ} \mathrm{C}$ ) before adding the polymerase. ${ }^{37}$ The intent of the approach is to eliminate or prevent generation of nonspecific PCR templates that may be synthesised at ambient temperature prior to thermal cycling. Specialized enzyme systems have been developed 
that inhibit the polymerase's activity at ambient temperature, either by the binding of an antibody ${ }^{38}$ or by the presence of covalently bound inhibitors that dissociate only after a high-temperature activation step. Hot-start/cold-finish PCR is achieved with new hybrid polymerases that are inactive at ambient temperature and are instantly activated at elongation temperature. ${ }^{13}$

\section{Miniprimer PCR}

It is a new PCR method that uses an engineered polymerase that can extend from miniprimers as short as 9 or 10 nucleotides. This method expands the scope of detectable sequences beyond those detected by standard methods using long primers and Taq polymerase. (39) Miniprimer PCR permits targeting to smaller primer binding regions, and is used to amplify conserved DNA sequences, such as the $16 \mathrm{~S}$ (or eukaryotic 18S) rRNA gene.

\section{Suicide PCR}

This type of PCR prescribes the use of single shot primers which avoids any risk of contamination and ensures absolute specificity. ${ }^{40}$ The term suicide is applied as any primer combination is used only once in a PCR.

Intersequence specific PCR, Nanoparticle-assisted PCR (nanoPCR), Overlap extension PCR, PAN-AC, Solid phase PCR, Thermal asymmetric interlaced PCR (TAIL-PCR) and Universal Fast Walking are some of the other advances in this field.

\section{CONCLUSION}

PCR can play an imperative role in diagnosing multitude of diseases with diverse and varied presentations. The speed and ease of use, sensitivity, specificity and robustness of PCR has revolutionised molecular biology and made PCR the most widely used and powerful technique with great spectrum of research and diagnostic applications.

\section{REFERENCES}

1. A new approach to the synthesis of polynucleotides: Khorana HG, Tener GM, Moffatt JG, Pol EH. Chem. Ind. London 1956,1523.

2. Mullis K, Faloona F, Scharf S, Saiki R, Horn G, Erlich H. Specific enzymatic amplification of DNA In vitro: the polymerase chain reaction. Cold Spring Harb Symp Quant Biol. 1986;51:263-73.

3. Mullis K. The unusual origin of the polymerase chain reaction. Sci Am. 1990;262:56-65.
4. Hill Philip $\mathbf{J}$ and Gordon SAB Stewart. The polymerase chain reaction in molecular and micro-biology. Biotechnology and Genetic Engineering Reviews 1992;10(1):343-78.

5. Saiki RK, Gelfand DH, Stoffel S, Scharf SJ, Higuchi R, Horn GT et al. Primer directed enzymatic amplification of DNA with a thermostable DNA polymerase. Science1988;239:487-91.

6. Engelke DR, Krikos A, Bruck ME and Ginsburg D. Purification of Thermus aquaticus DNA polymerase expressed in Escherichia coli. Analytical Biochemistry1990;191:396-400.

7. Pluthero FG. Rapid purification of high-activity Taq DNA polymerase. Nucleic Acids Res. 1993;21(20):4850-51.

8. Wolff K, Schoen ED, Peters-Van Rijn J. Optimizing the generation of random amplified polymorphic DNAs in chrysanthemum. Theor. Appl. Genet. 1993;86:1033-7.

9. Birren B, Green ED, Klapholz S, Myers RM, Roskams J, eds. Genome Analysis: A Laboratory Manual. Analyzing DNA. Cold Spring Harbor Laboratory Press, Cold Spring Harbor, NY, 1997, Vol. 1.

10. Burgmann H, Pesaro M, Widmer F, Zeyer J. A strategy for optimizing quality and quantity of DNA extracted from soil.J Microbiol Methods 2001;45(1):7-20.

11. Roose-Amsaleg CL, Garnier-Sillam E, Harry M. Extraction and purification of microbial DNA from soil and sediment samples. Appl Soil Ecol 2001;18:47-60.

12. Yang $S$ and Rothman RE. PCR-based diagnostics for infectious diseases: uses, limitations, and future applications in acute-care settings. Lancet 2004;4(6):337-348.

13. Rahman MT, Uddin MS, Sultana R, Moue A, Setu M. Polymerase Chain Reaction (PCR): A Short Review. AKMMC J 2013;4(1):30-36

14. Lijun Wang, Haitong Gu, Xinxin Lu. A rapid low-cost realtime PCR for the detection of klebsiella pneumonia carbapenemase genes. Ann Clin Microbiol Antimicrob 2012;11:9.

15. Tang YW, Procop GW, Persing DH . Molecular diagnostics of infectious diseases. Clin Chem 1997;43(11):2021-38.

16. Clement $\mathrm{J}$ and Heyman P. PCR for diagnosis of viral infections of the central nervous system. Lancet 1997;349:1256

17. Pearson $\mathrm{H}$ and Stirling D. Cloning and Mutagenesis. A Technical Overview. In: Methods in Molecular Biology, PCR Protocols. John M. S. Bartlett and David Stirling (Eds), $2^{\text {nd }}$ ed, 2003, Vol 226, Pg 467-8. Humana Press, Totowa, New Jersey, USA.

18. Grunenwald H. Optimization of Polymerase Chain Reactions. In: Methods in Molecular Biology, PCR Protocols. Bartlett John MS and Stirling D (Eds), $2^{\text {nd }}$ ed, 2003, Vol 226, Pg 89-100. Humana Press, Totowa, New Jersey, USA.

19. Roux KH. Optimization and troubleshooting in PCR.Cold Spring Harb Protoc; 2009;4(4):1-6.

20. Jackson CR, Fedorka Cray PJ, Barret JB. Use of a genus and species specific multiplex PCR for amplification of Enterococci. J Clin Microbiol 2004;42:3558-65.

21. Templeton KE, Scheltinga SA, Sillekens P, Crielaard JW, Van Dam AP, Goenssens H, et al. Development and clinical evaluation of an internally controlled, single tube multiplex real time PCR assay for detection of Legionella 
pneumophila and other Legionella species. J Clin Microbiol 2003;41:4016-21.

22. Jann-Yuan W, Li-Na N, Chin-Sheng C, Chung-Yi H, ShuKuan W, Hsin-Chih L, et al. Performance assessment of a Nested-PCR assay and the BD ProbeTec ET system for detection of Microbacterium tuberculosis in clinical specimens. J Clin Microbiol 2004;42:4599-603.

23. Kitagawa $\mathrm{Y}$, Ueda $\mathrm{M}$, Ando $\mathrm{N}$, Endo $\mathrm{M}$, Ishibiki $\mathrm{K}$, Kobayashi Y. Rapid diagnosis of methicillin-resistant Staphylococcus aureus bacteremia by Nested Polymerase Chain Reaction. Ann Surg 1996;224:665-71.

24. Ke D, Menard C, Picard FJ, Boissinot M, Ouellette M, Roy $\mathrm{PH}$. Development of conventional and Real time PCR assays for the rapid detection of group B Streptococci. Clin Chem 2000;46:324-31.

25. Moretti T, Koons B, Budoele B. Enhancement of PCR amplification yield and specificity using AmpliTaq Gold DNA polymerase. Biotechniques 1994;16:1134-7.

26. Salomon RN. Introduction to quantitative reverse transcriptase polymerase chain reaction. Diag Mol Pathol 1995;4:82-4.

27. Demeke T, Jenkins GR. Influence of DNA extraction methods, PCR inhibitors and quantification methods on realtime PCR assay of biotechnology-derived traits. Anal Bioanal Chem 2010;396(6):1977-90.

28. Mirhendi H, Diba K, Rezaei A, Jalalizand N, Hosseinpur L, Khodadadi H. Colony PCR is a rapid and sensitive method for DNA amplification in yeasts. Iranian Journal of Public Health 2007;36(1):40-4.

29. Packeiser H, Lim C, Balagurunathan B, Wu J, Zhao H. An extremely simple and effective colony PCR procedure for bacteria, yeasts and micro algae. Applied Biochemistry and Biotechnology 2013;169(2):695-700.

30. Darawi MN, Ai-Vyrn C, Ramaswamy K, Hua POJ, Pin TM, Kamaruzzaman SB, et al. Allele specific polymerase chain reaction for detection of Alzheimer's disease related single nucleotide polymorphisms. BMC Med Genet 2013;14(1):18 .

31. Stemmer WPC, Crameri A, Ha KD, Brennan TM, Heyneker HL. Single step assembly of a gene and entire plasmid from large numbers of oligodeoxyribonucleotides. Gene 1995;164(1):49-53.

32. Citaratan M, Tang TH, Tan SC, Hoe CH, Saini R, Tominaga $\mathrm{J}$, et al. Asymmetric PCR for good quality ssDNA generation towards DNA aptamer production. Songklanakarin J Sci Technol 2012;34(2):125-31.

33. Pierce KE, Sanchez JA, Rice JE, Wangh LJ. Linear-afterthe-exponential (LATE)-PCR: Prime design criteria for high yields of specific single-stranded DNA and improved realtime detection. Proc Natl Acad Sci USA 2005;102(24):8609-14.

34. Pierce KE, Wangh LJ. Linear-after-the-exponential polymerase chain reaction and allied technologies. Realtime detection strategies for rapid, reliable diagnosis from single cells. Methods Mol Med 2007;132:65-85.

35. Schwartz JJ, Lee C, Shendure J. Accurate gene synthesis with tag-directed retrieval of sequence-verified DNA molecules. Nat Methods 2012;9(9):913-5.

36. Vincent $\mathrm{M}, \mathrm{Xu} \mathrm{Y}$, Kong $\mathrm{H}$. Helicase-dependent isothermal DNA amplification. EMBO Rep 2004;5(8):795-800.

37. Chou Q, Russell M, Birch DE, Raymond J, Bloch W. Prevention of pre-PCR mis-priming and primer dimerization improves low-copy-number amplifications. Nucleic Acids Res 1992;20(7):1717-23.

38. Kellogg DE, Rybalkin I, Chen S, Mukhamedova N, Vlasik T, Siebert PD, et al. TaqStart Antibody: "hot start" PCR facilitated by a neutralizing monoclonal antibody directed against Taq DNA polymerase. Biotechniques 1994;16(6):1134-7.

39. Isenbarger TA, Finney M, Rios-Velazquez C, Handelsman J, Ruvkun G. Miniprimer PCR, a new lens for viewing the microbial world. Appl Environ Microbiol 2008;74(3):840-9.

40. Raoult D, Aboudharam G, Crubezy E, Larrouy G, Ludes B, Drancourt M. Molecular identification by "suicide PCR" of Yersinia pestis as the agent of Medieval black death. Proc Natl Acad Sci USA 2000,97(23):12800-3. 